\section{AB0324 REASONS FOR DISCONTINUATION OF BIOLOGICS IN PATIENTS WITH REFRACTORY RHEUMATOID ARTHRITIS: RESULTS OF A RETROSPECTIVE STUDY}

V. Sygyrta ${ }^{1}$, S. Glukhova ${ }^{1}$, E. Aronova ${ }^{1}$, A. Satybaldyev ${ }^{1}$, A. Lila ${ }^{1} .{ }^{1}$ V.A. Nasonova Research Institute of Rheumatology, Moscow, Russian Federation

Background: Refractory rheumatoid arthritis (RRA) is a subtype of rheumatoid arthritis (RA), in which the sequential administration of optimal methotrexate (MT) doses in combination with glucocorticoids (GCs), and at least - two biologic disease-modifying antirheumatic drugs (bDMARDs) with different mechanisms of action during 18-24 months does not lead to a significant decrease in the inflammatory activity of RA.

Objectives: Analysis of the selection strategy and the "survival" of bDMARDs in patients with RRA.

Methods: The retrospective study included data of 95 RRA patients ( 80 females, $80.8 \%$ ), aged 23 to 80 years (mean age 57 years), treated with bDMARDs. Mean RA duration was $11.9 \pm 7.6$ years. All patients were divided into 6 groups depending on the number of the lines of therapy (LOTs) received (from 2 to 7 consecutive bDMARDs). Totally 348 cases of bDMARDs administration were analyzed.

Results: TNF-a inhibitors were most commonly used as the first and second lines of therapy: infliximab (INF) - 43 prescriptions, adalimumab (ADA) - 39, etanercept (ETC) - 25, certolizumab pegol (CZP) - 11, golimumab (GLM) - 6. Abatacept (ABA) was prescribed in 32 cases, rituximab (RTM) in 22 cases, and tocilizumab (TCZ) - in 12 cases. The following reasons for bDMARDs discontinuation were identified: lack of efficacy (LE) (55.2\% of cases), adverse events (AE), including serious adverse events ( $14.8 \%$ of cases), administrative reasons ( $10.0 \%$ of cases), persistent remission $(2.1 \%$ of cases), pregnancy $(0.6 \%)$, and other ( $17.3 \%$ of cases)

TNF- $a$ inhibitors were also used in third-line bDMARDs therapies, but preference was given to drugs with a different mechanism of action: ABA-20 patients (23.2\%), RTM - 20 (21.1\%), TCZ - 15 (15.8\%), ETC - 22 (23.2\%), ADA - 9 $(9.5 \%)$, INF $-4(4.1 \%)$, CZP $-3(3.1 \%)$. Treatment was discontinued in 74 patients $(77.9 \%)$. In this cohort the following reasons for bDMARDs withdrawal were identified: LE $(54.1 \%), \mathrm{AE}(17.6 \%)$, administrative reasons $(9.5 \%)$, remission $(1.3 \%)$, and other (17.5\%).

The fourth line of therapy was administered in 45 patients: ABA-16 (35.6\%), RTM-9 (20\%), ADA - 6 (13.3\%), TCZ - 5 (11.1\%), ETC - 4 (8.9\%), CZP - 4 (8.9\%), GLM -1 (2.2\%). The reasons for discontinuation (29 patients, $64.4 \%$ ) were as follows: LE (44.8\%), AE (13.8\%), other $(41.4 \%)$.

The fifth bDMARDs was used in 3 patients: TCZ-6 (46.1\%), RTM - $3(23.1 \%)$, ABA - $1(7.7 \%)$, ETC - 1 (7.7\%), ADA - 1 (7.7\%), GLM - 1 (7.7\%). Therapy was discontinued in 11 patients $(84.6 \%)$ for the following reasons: LE (45.5\%), AE $(18.2 \%)$, other $(36.3 \%)$

The sixth line of therapy was necessary in 4 patients: ETC $(25 \%)$, GLM $(25 \%)$, CZP $(25 \%)$, ABA $(25 \%)$ and was discontinued in $3(75 \%)$ of them due to $\mathrm{AE}$ (33.3\%) and other reasons (66.7\%). One patient received TCZ as the seventh line of therapy.

Mean duration of the first line of therapy was $7.6 \pm 6.5$ months, of the second line - $9.6 \pm 7.5$ months, of the third line - $11.5 \pm 7.1$ months, fourth line $-12.5 \pm 8$ months, fifth line $-13.4 \pm 4.8$ months, and sixth line $-14.6 \pm 4.4$ months. Statistical analysis revealed significant differences $(p<0.05)$ in the mean duration of therapy (retention on therapy) between the 1st and 3rd, as well as the between the $1 \mathrm{st}$ and 4 th lines of therapy. There were no significant differences in rates of bDMARDs discontinuation due to LE or AE. The rates of bDMARDs discontinuation did not differ significantly in the study groups.

Conclusion: The mean retention on a drug in the 3rd and 4th lines of therapy in patients with refractory rheumatoid arthritis was significantly longer than on the 1st line of therapy. The most common reason for bDMARDs discontinuation was lack of efficacy. Additional lines of bDMARDs therapy were not associated with increasing rates of adverse events.

Disclosure of Interests: None declared

DOI: 10.1136/annrheumdis-2020-eular.2735

\section{AB0325 FLUORESCENCE OPTICAL IMAGING (FOI) AIDS DIFFERENTIAL DIAGNOSIS OF RHEUMATIC DISEASES AND INCREASES TREATMENT RESPONSE RATE IN RA THROUGH PATIENT STRATIFICATION}

M. Ferl ${ }^{1}$, S. Ohrndorf ${ }^{2}$, J. Berger ${ }^{3}$, A. Briel ${ }^{3}$, P. Welker ${ }^{2} .{ }^{1}$ Medical Faculty University Hospital Magdeburg, Berlin, Germany; ${ }^{2}$ Charité - University Medicine Berlin, Berlin, Germany; ${ }^{3}$ Xiralite GmbH, Berlin, Germany

Background: In recent years, indocyanine green (ICG)-enhanced FOI has become clinically available as a novel tool for the early detection of rheumatic diseases, assessment of disease activity and monitoring of treatment response. The high sensitivity of this method allows visualization of slight changes in the microcirculation of the hands as a sign of inflammation. Different rheumatic diseases present characteristic signal enhancement patterns, which may facilitate differential diagnosis. Signal enhancement in metacarpophalangeal (MCP) joints, for example, can frequently be seen in patients with rheumatoid arthritis (RA).

Objectives: We analyzed data of a multicentric clinical study (OPERA, $n=3300$ ) including patients with different rheumatic diseases. Patients were divided into groups using clinical parameters followed by FOI examination to test the hypothesis that this method can improve the diagnosis.

Methods: The present study involved 200 patients with RA ( $n=200)$, divided into groups according to Steinbrocker's (STBR) staging system, patients that had degenerative osteoarthritis $(O A, n=100)$, and a control group with out clinical symptoms $(n=40)$. RA patients were examined before and during treatment with biologicals, glucocorticoids (GK), or DMARDs. Clinical and laboratory assessments were made by analyses of DAS28, patient questionnaires, rheumatoid factor (RF), anti-citrullinated protein antibodies (ACPA), erythrocyte sedimentation rate (ESR), and x-rays. FOI signal intensity (SI) was defined by ratio of areas with $\mathrm{SI}$ in patients and controls. Image sequences were analyzed visually, and MCP joints were judged as positive if in the early phase of ICG inflow, a higher SI in any MCP region was found in comparison to the control group.

Results: Initially, serum factors typical for RA patients were analyzed in the different groups. In $23 \%$ of OA patients RF and/or ACPA were detected in the serum. Surprisingly, in the STBR I group, only $35 \%$ of patients were tested as serum-positive for RF and/or ACPA. After FOI, the patients were subdivided into two groups with and without ICG enrichment in MCPs. In the MCP-positive group, the percentage of RF/ACPA-positive STBRI patients increased to $83 \%$, with only $25 \%$ seropositive patients in the MCP-negative group. In STBRII-IV cohorts, the proportion of RF/ACPA-positive patients was initially higher as in the STBRI group, but also increased after FOI analysis of MCP positivity. In the group treated with biologicals (STBRI-IV), responders were identified both by clinical parameters and $\mathrm{FOI}$. After treatment, $42 \%$ of all analyzed patients were found to respond to treatment. Compared to all patients, the MCP-positive group showed a significantly increased response rate at $71 \%$, while all patients $(100 \%)$ in the MCP-negative group were identified as non-responders (Figure 1).

Conclusion: The study indicates that $\mathrm{FOI}$ is highly effective for the diagnosis of RA, selection of the appropriate therapy, and for the monitoring of therapeutic success. Treatment response rate can be increased (from $42 \%$ to $71 \%$ ) through patient stratification in terms of ICG enrichment in MCP. Figure.
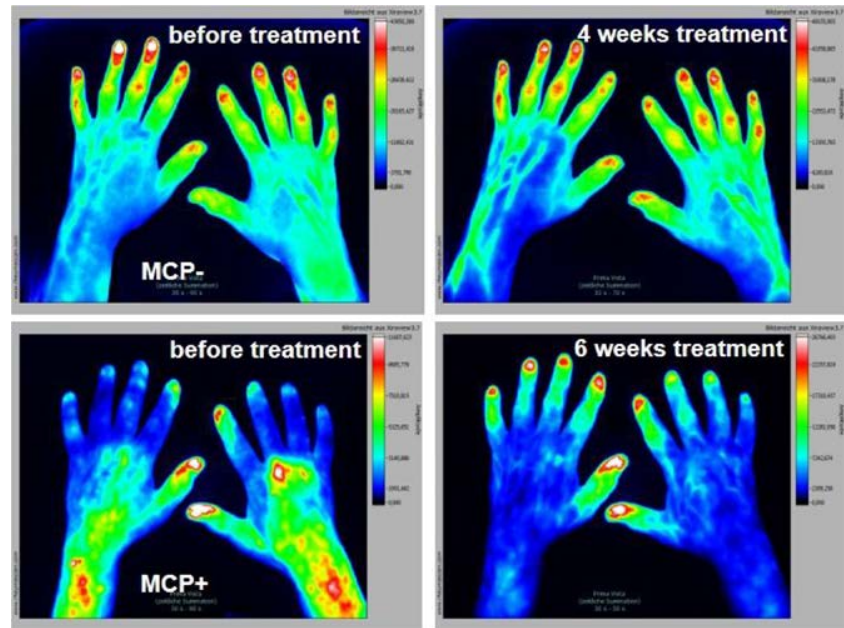

Figure 1: Examples of $\mathrm{FOl}$ images in patients with suspected early rheumatoid arthritis before and after treatment with anti-rheumatic biologics

Disclosure of Interests: Mark Ferl: None declared, Sarah Ohrndorf: None declared, Jörn Berger Employee of: Xiralite $\mathrm{GmbH}$, Andreas Briel Shareholder of: Xiralite $\mathrm{GmbH}$, Pia Welker Employee of: Xiralite $\mathrm{GmbH}$ DOI: 10.1136/annrheumdis-2020-eular.4332 